\title{
Oscillation parameters of red giants observed in the $\operatorname{CoRoT}$ exofield
}

\author{
N. Themessl ${ }^{1}$, T. Kallinger ${ }^{1}$, J. Montalbán ${ }^{2}$, and R. A. García ${ }^{3}$ \\ ${ }^{1}$ Institute for Astrophysics, University of Vienna, \\ Türkenschanzstraße 12, A-1180 Vienna, Austria \\ email: nathalie.themessl@univie.ac.at \\ ${ }^{2}$ Institut d'Astrophysique et de Géophysique, Université de Liège, Belgium \\ ${ }^{3}$ Laboratoire AIM, CEA/DSM-CNRS, Université Paris 7 Diderot, IRFU/SAp, France
}

\begin{abstract}
The high-precision data obtained by the CoRoT (Convection, Rotation and planetary Transits) space mission allows firm detections of solar-like oscillations in a great variety of different stars. We derived reliable estimates of the frequency of maximum oscillation power $\nu_{\mathrm{max}}$ and the large frequency separation $\Delta \nu$ of more than $300 \mathrm{cool}$ red giants, that were observed by CoRoT. A detailed study of their seismic parameters provides an exclusive view of the population of the observed giants.
\end{abstract}

Keywords. stars: late-type, stars: oscillations, stars: fundamental parameters

\section{Introduction}

After the depletion of hydrogen in their cores, stars reach the giant branch. In these cool $\mathrm{G}$ and $\mathrm{K}$ stars solar-like oscillations are expected with long periods in the range of hours to several days. They exhibit radial modes of high radial order and nonradial mixed $\mathrm{p} / \mathrm{g}$ modes, which reveal important information about their innermost structure. Nowadays, many different methods allow the analysis of these very complex spectra including the extraction of individual frequencies (Mosser et al. 2012). However, long continuous observations over several years are necessary to properly resolve the oscillations and to enable unambiguous detections. Just recently, space missions such as CoRoT (Baglin et al. 2006) and Kepler have provided the necessary data accuracy, which allows to probe and understand the oscillation spectra of these evolved stars.

\section{Data and observation}

In this project, we use more than 450 red-giant targets, that were observed by the CoRoT space telescope. The available data consist of reduced chromatic and monochromatic lightcurves that were obtained during the first (LRc01) and the second long run (LRa01) of the satellite.

\section{Global oscillation parameters}

The power spectrum of solar-like oscillations shows a white noise component, a frequency-dependent background component modified by several super-Lorentzian functions (with the exponent equal to 4 instead of 2) and a power excess generated through pulsation, whose shape can be approximated by a Gaussian (Fig. 1). These characteristic features defined our global model, which was used to derive the oscillation parameters. 

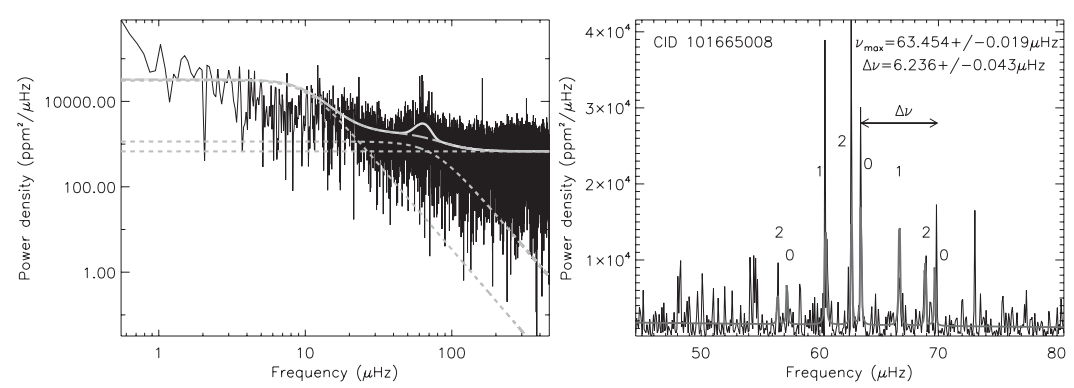

Figure 1. Left: The power density spectrum including a global fit to the spectrum with (solid yellow) and without (large dashed blue) a Gaussian, and the three different background components (dashed green). Right: The residual power density spectrum of the same star with the red line characterizing the best model fit, which is used to derive the p-mode spacings.

After the visual inspection of each individual power spectrum about 100 red-giant targets were excluded from the analysis due to the lack of a distinctive power excess hump. Following Kallinger et al. $(2010,2012)$, we fitted the global model to the observed power density spectrum by using a Bayesian nested sampling algorithm (MultiNest; Feroz et al. 2009). Besides characterizing the granulation background signal, the global fit gives the frequency of maximum oscillation power $\nu_{\max }$ (i.e. the centre of the oscillation power excess). The oscillation power excess itself consists of a sequence of near regularly spaced radial and nonradial modes. Most importantly, these regularities can be characterized by the so-called large and small frequency separation $\Delta \nu$ and $\delta \nu$, respectively, which represent the frequency difference between consecutive modes with the same spherical degree and consecutive modes but with a difference in spherical degree of two. In order to derive $\Delta \nu$ and $\delta \nu$ we fitted a sequence of Lorentzian profiles to the central part of the power excess, again using MultiNest. The advantage of our approach is that MultiNest scans the parameter space for a real global maximum in the likelihood "mountains" and delivers a very robust solution preventing us from over-fitting the data.

\section{Discussion}

The firm detection of solar-like oscillations in many different stars would not only provide a better understanding of the characteristics of stochastically excited oscillations, but would also improve current state-of-the-art stellar structure and evolution models. We analyzed a large number of red-giant stars and derived the frequency of maximum oscillation power, the large frequency separation and the mode degree. Since both seismic parameters scale with the stellar mass, radius, and effective temperature (Kjeldsen \& Bedding 1995), they can be further used to determine the fundamental parameters of a star. As these parameters change with stellar evolution, they provide crucial insights into the population of the observed giants (Miglio et al. 2012).

\section{References}

Baglin, A., Auvergne, M., Barge, P., et al. 2006, ESA SP-1306, 33

Feroz, F., Hobson, M. P., \& Bridges, M. 2009, MNRAS, 398, 1601

Kallinger, T., Hekker, S., Mosser, B., et al. 2012, A\& A, 541, A51

Kallinger, T., Mosser, B., Hekker, S., et al. 2010, A\&A A, 522, A1

Kjeldsen, H. \& Bedding, T. R. 1995, A\&\&A, 293, 87

Miglio, A., Brogaard, K., Stello, D., et al. 2012, MNRAS, 419, 2077

Mosser, B., Elsworth, Y., Hekker, S., et al. 2012, AछA, 537, A30 